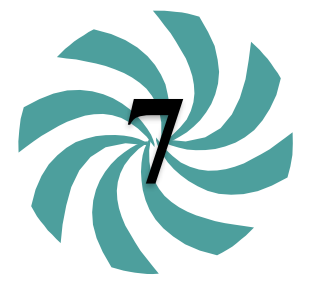

Tecnociencia, Vol. 23, $\mathrm{N}^{\circ} 1: 125-142$

Enero-Junio 2021

\title{
BIOMETRÍA DE Lutjanus peru (PARGO SEDA) y Lutjanus guttatus (PARGO DE LA MANCHA) (PISCES: LUTJANIDAE) EN EL GOLFO DE MONTIJO, PACÍFICO DE PANAMÁ.
}

\author{
1 Valerio A. Gómez C., (iD) ${ }^{2}$ Benjamín Sánchez V. (iD \& $\mathbf{3}^{3} \mathbf{J} u a n$ \\ A. Gómez H. \\ ${ }^{1}$ Universidad Interamericana de Panamá, Programa de Maestría en Gestión Ambiental, \\ e-mail: valeriogomez2013@gmail.com \\ ${ }^{2}$ Autoridad de los Recursos Acuáticos de Panamá, Regional de Veraguas, e-mail: \\ benji0386.bs@gmail.com \\ ${ }^{3}$ Universidad de Panamá, Departamento de Biología Marina y Limnología; \\ juanay05@hotmail.com
}

\section{RESUMEN}

Existe una presión pesquera sobre dos especies, Lutjanus peru y Lutjanus guttatus en el Golfo de Montijo, del Pacífico veragüense. Entre noviembre 2013 y septiembre de 2018, semanalmente se registró dos especies de interés comercial; se determinó y ana lizó el volumen de desembarque, la talla y el peso y se correlacionaron. En L. peru las tallas oscilaron entre 12 y $84 \mathrm{~cm}$ con promedio de $40,7 \mathrm{~cm}$; el peso entre los 0,22 $\mathrm{kg}$ y $6,35 \mathrm{~kg}$ con promedio de $1,33 \mathrm{~kg}$; el volumen totalfue $169132 \mathrm{~kg}$, un r $=0,76 \mathrm{y}$ $\mathrm{R} 2=0,5705$. La talla de L. guttatus osciló en los 20 y $79 \mathrm{~cm}$ con promedio de $38,5 \mathrm{~cm}$ y el peso entre $0,22 \mathrm{~kg}$ y $2,04 \mathrm{~kg}$ con el promedio $0,6 \mathrm{~kg}$; el volumen reportado fue $174002 \mathrm{~kg}, \mathrm{r}=0,73$. R2=0,5338. Las capturas de $L$. peru representaron el 49,29\%; mientras que para $L$. guttatus correspondió a $50,71 \%$. La relación talla-peso para ambas especies fue significativa.

PALABRAS ClaVES

Lutjanus peru, Lutjanus guttatus, biometría, presión pesquera. 
BIOMETRY OF Lutjanus peru (PACIFIC RED SNAPPER) AND Lutjanus guttatus (SPOTTED ROSE SNAPPER) (PISCES: LUTJANIDAE) IN THE GULF OF MONTIJO, PANAMA PACICIF.

\begin{abstract}
There is fishing pressure on two species, Lutjanus peru and Lutjanus guttatus in the Gulf of Montijo, in Veraguas Pacific. Between November 2013 and September 2018, two species of commercial interest were registered weekly. The volume of Lutjanus peru and Lutjanus guttatus, sizes and weight were determined and analyzed, and the sizes were correlated with the weights, obtaining the determination coefficients (R2) and the correlation coefficients (r). In L. peru the sizes ranged between 12 and $84 \mathrm{~cm}$ with an a verage of $40.7 \mathrm{~cm}$; the weight between $0.22 \mathrm{~kg}$ and $6.35 \mathrm{~kg}$ with an average of $1.33 \mathrm{~kg}$; the total volume was $169,132 \mathrm{~kg}, \mathrm{R} 2=0.5705, \mathrm{r}=0.76$. The size of $L$. guttatus was between 20 and $79 \mathrm{~cm}$, with an average of $38.5 \mathrm{~cm}$ and the weight between $0.22 \mathrm{~kg}$ and $2.04 \mathrm{~kg}$ with the avera ge $0.6 \mathrm{~kg}$; the reported volume was 174,002 $\mathrm{kg}, \mathrm{R} 2=0.5338, \mathrm{r}=0.73$. L. peru represented $49.29 \%$ of the catches; on the other hand, L. guttatus corresponded to $50.71 \%$. The length-weight relationship for both species was significant.
\end{abstract}

\title{
KEYWORDS
}

Lutjanus peru, Lutjanus guttatus, biometry, fishing pressure.

\section{INTRODUCCIÓN}

El recurso pesquero es uno de los productos alimentarios más comercializados del mundo, la mitad del valor de las exportaciones pesqueras procede de países en desarrollo (FAO, 2016). La costa del Pacífico panameño con una extensión de $1700 \mathrm{~km}$ de longitud aproximadamente, es heterogénea y se extiende desde el Este en la Provincia de Darién, hasta Punta Burica en el Oeste (Maté, 2005). En ella encontramos una gran diversidad marina de peces, y algunas de estas especies son de importancia comercial y alimenticia.

El litoral Pacífico de la Provincia de Veraguas, por ser una zona de interés pesquero, se ha convertido en un área importante para la descripción de la biodiversidad marina (Vega \& Villarreal, 2003). En el Golfo de Montijo se realiza una importante extracción de recurso pesquero entre ellos, las especies de interés comercial, Lutjanus guttatus, Lutjanus peru, Lutjanus jordani, que corresponden a la familia Lutjanidae (CREHO, 2009). 
La Familia Lutjanidae, conocidas como "Pargos", son de diversos tamaños y alcanzan hasta $1,7 \mathrm{~m}$ de longitud total en la especie Lutjanus novemfasciatus, $L$. peru alcanza los $95 \mathrm{~cm}$ de longitud total (LT) y $L$. guttatus alcanza los $80 \mathrm{~cm}$ de LT (ARAP, 2011). Existen 17 géneros de esta familia, distribuidos en los mares tropicales; la mayoría habita en la región Indo-Pacífico, el género Lutjanus es el más numeroso con 67 especies, incluye nueve del Pacífico Oriental y 12 con 4 géneros en el Pacífico Panameño (Robertson \& Allen, 2015). La mayoría viven en aguas someras, aunque algunas se encuentran entre los 100 y $500 \mathrm{~m}$ de profundidad (Lucano-Ramírez et al. 2012).

Las investigaciones escasas, logística deficiente y censos de capturas incompletos se han convertido en una dificultad para monitorear el recurso a lo largo del Golfo de Montijo, aunque en el área existen algunos registros de abundancia de esta pesquería. En ese sentido investigaciones sobre el ciclo biológico, abundancia, tallas de capturas y otros aspectos relevantes de estas especies, ayudan a la toma de decisiones para el manejo sostenible y responsable del recurso, de allí la importancia de analizar las relaciones biométricas de Lutjanus peru (pargo seda) y Lutjanus guttatus (pargo de la mancha), especies de interés comercial en el Pacifico veragüense.

\section{MATERIALES Y MÉTODOS Área de estudio}

El Golfo de Montijo posee una extensión de $894,52 \mathrm{~km}^{2}$ (Maté, 2005), se localiza entre los distritos de Montijo, Soná, Río de Jesús, Mariato y Santiago, provincia de Veraguas e incluye toda la porción costeromarina del Golfo de Montijo y la isla Leones (Fig. 1). 


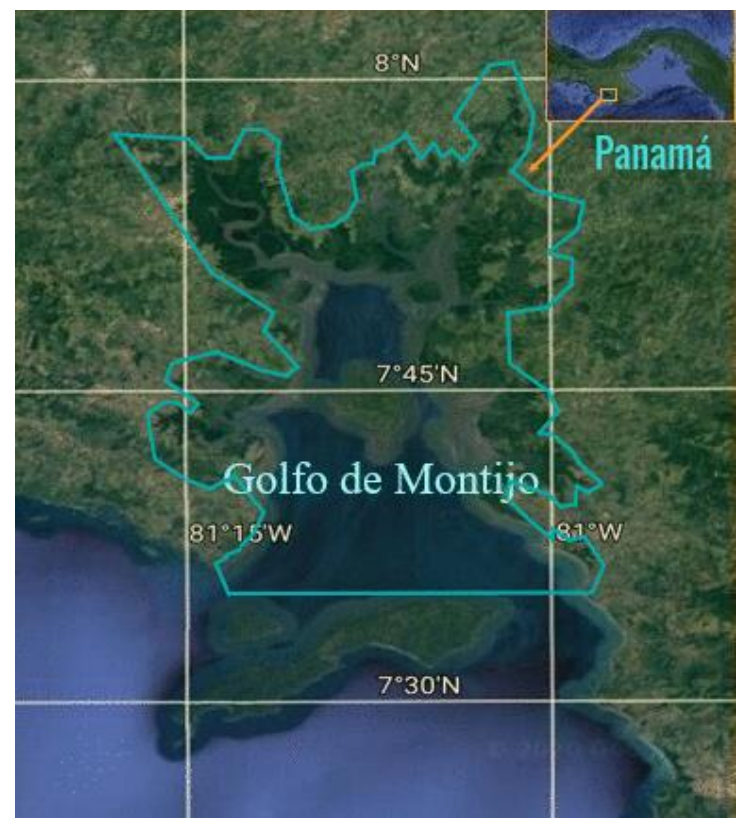

Fig. 1 Golfo de Montijo, Pacifico de Veraguas, Panamá

La mayor parte del sitio Ramsar corresponde a bosque de manglar con áreas inundadas, estuarios, playas y arrecifes de corales (CREHO, 2009). En las desembocaduras de estos ríos se distribuyen los manglares del Golfo de Montijo (Cámara et al. 2004).

\section{Muestreos}

Las muestras se obtuvieron de la pesca artesanal desembarcadas en Puerto Mutis, durante el periodo 2013-2018. Las mismas fueron identificadas mediante la Guía de Peces de Interés Comercial para el Pacífico de Panamá (ARAP, 2011). Se utilizó el Sistema de Información en línea de Peces Costeros del Pacífico Oriental Tropical para corroborar la identificación (Robertson \& Allen, 2008, 2015).

Se analizaron 1144 muestras aleatorias de dos especies de interés comercial, de los cuales 810 de L. peru y 334 de L. guttatus.

Se determinó el volumen de capturas, talla, peso, y se hicieron las correlaciones correspondientes. El peso de las capturas fue determinado en libras con una báscula Insta-matica con capacidad de 40 lbs x 1 onza de precisión y transformada a $\mathrm{kg}$. 
A los ejemplares seleccionados, se le midió la longitud total (LT) con un ictiómetro flexible modelo C-THRU en centímetros y pesados con una balanza digital con $0,1 \mathrm{~g}$ de precisión.

Los datos fueron registrados en los formularios de muestreo biológicopesquero, ARAP/OSPESCA, para los análisis biométricos.

\section{Análisis de los resultados:}

El volumen, peso y talla procede de datos de la serie de tiempo de la Autoridad de los Recursos Acuáticos (ARAP), entre los periodos de 2013 al 2018, en temporadas secas y lluviosas, provenientes de los puertos de desembarques de Montijo, Soná, Río de Jesús, Mariato y Las Palmas. Las mediciones talla y peso, se realizaron en Puerto Mutis.

Se analizó el volumen de captura de cada especie. Los datos de talla máxima, mínima y promedio, fueron tratados con histogramas de frecuencia y agrupados por ámbito de talla de cada especie. El peso promedio de las especies se obtuvo de la suma total de los pesos comprendidos entre los años 2015 - 2018. Se determinó la relación talla - peso mediante correlación.

\section{RESULTADOS}

\section{Volumen de captura}

La especie L. peru registró un total de $169132 \mathrm{~kg}$, que corresponde a 49, $29 \%$ del total de captura en el estudio. El volumen osciló entre las $10456 \mathrm{~kg}$ y $45896 \mathrm{~kg}$, este último en el año 2017 y el volumen promedio de $28188 \mathrm{~kg}$ (Fig. 2).

Se reportó un total de $174002 \mathrm{~kg}$, correspondiente a 50,71\% del total de captura de L. guttatus, el volumen osciló entre 9699 y $75488 \mathrm{~kg}$, este último el mayor reportado para esta especie en el año 2015, con un volumen promedio de $29000 \mathrm{~kg}$ (Fig. 2). 


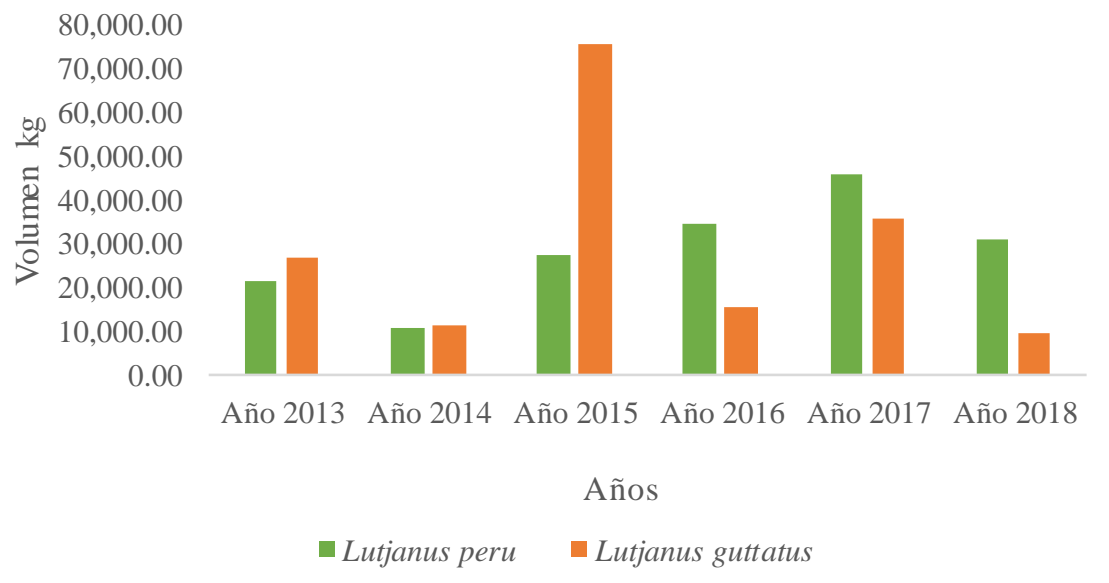

Fig. 2 Volumen de captura de Lutjanus peru y Lutjanus guttatus en el Golfo de Montijo, periodo 2013-2018

\section{Talla y peso}

La talla de $L$. peru osciló entre 12 y $84 \mathrm{~cm}$, con un promedio total de $40,7 \mathrm{~cm}$, talla media de captura y en L. guttatus varió entre 20 y $79 \mathrm{~cm}$, con promedio de captura de 38,5 cm (Cuadro 1).

Cuadro. 1 Talla (máxima, promedio, mínima) de las especies Lutjanus peru y Lutjanus guttatus, Golfo de Montijo 2013-2018

\begin{tabular}{llllll}
$\boldsymbol{\downarrow}$ Especie/ Años $\rightarrow$ & $\mathbf{2 0 1 3 / 1 4}$ & $\mathbf{2 0 1 5}$ & $\mathbf{2 0 1 6}$ & $\mathbf{2 0 1 7}$ & $\mathbf{2 0 1 8}$ \\
\hline Lutjanus peru & & & & & \\
\hline Talla máxima & $52 \mathrm{~cm}$ & $68 \mathrm{~cm}$ & $74 \mathrm{~cm}$ & $\mathbf{8 4 ~ c m}$ & $78 \mathrm{~cm}$ \\
\hline Talla promedio & $34,62 \mathrm{~cm}$ & $41 \mathrm{~cm}$ & $38 \mathrm{~cm}$ & $43 \mathrm{~cm}$ & $47 \mathrm{~cm}$ \\
\hline Talla mínima & $\mathbf{1 2} \mathbf{~ c m}$ & $20 \mathrm{~cm}$ & $13 \mathrm{~cm}$ & $26 \mathrm{~cm}$ & $24 \mathrm{~cm}$ \\
\hline Lutjanus guttatus & & & & & \\
\hline Talla máxima & $43 \mathrm{~cm}$ & $\mathbf{7 9} \mathrm{cm}$ & $53 \mathrm{~cm}$ & $54 \mathrm{~cm}$ & $78 \mathrm{~cm}$ \\
\hline Talla promedio & $36,77 \mathrm{~cm}$ & $38 \mathrm{~cm}$ & $39 \mathrm{~cm}$ & $37 \mathrm{~cm}$ & $42 \mathrm{~cm}$ \\
\hline Talla mínima & $25 \mathrm{~cm}$ & $22 \mathrm{~cm}$ & $25 \mathrm{~cm}$ & $\mathbf{2 0} \mathbf{c m}$ & $28 \mathrm{~cm}$
\end{tabular}

El peso de $L$. peru varió entre 0,22 y $6,35 \mathrm{~kg}$ con promedio de $1,01 \mathrm{~kg}$; mientras que para L. guttatus el peso estuvo entre 0,22 y $2,04 \mathrm{~kg}$, con media de 0,63 kg. (Cuadro 2). 
Cuadro. 2 Peso (máximo, promedio, mínimo) de las especies Lutjanus peru y Lutjanus guttatus, Golfo de Montijo 2013-2018

\begin{tabular}{lccccc}
$\downarrow$ Especie/ Años $\rightarrow$ & $\mathbf{2 0 1 3 / 1 4}$ & $\mathbf{2 0 1 5}$ & $\mathbf{2 0 1 6}$ & $\mathbf{2 0 1 7}$ & $\mathbf{2 0 1 8}$ \\
\hline $\begin{array}{l}\text { Lutjanus peru } \\
\text { Peso máximo }\end{array}$ & $\mathrm{S} / \mathrm{R}$ & $3,4 \mathrm{~kg}$ & $1,58 \mathrm{~kg}$ & $\mathbf{6 , 3 5} \mathbf{~ k g}$ & $4,53 \mathrm{~kg}$ \\
\hline Peso promedio & $\mathrm{S} / \mathrm{R}$ & $1,2 \mathrm{~kg}$ & $0,68 \mathrm{~kg}$ & $1,04 \mathrm{~kg}$ & $1,12 \mathrm{Kg}$ \\
\hline Peso mínimo & $\mathrm{S} / \mathrm{R}$ & $\mathbf{0 , 2 2} \mathbf{~ k g}$ & $0,22 \mathrm{~kg}$ & $0,22 \mathrm{~kg}$ & $0,45 \mathrm{~kg}$ \\
\hline Lutjanus guttatus & & & & & \\
\hline Peso máximo & $\mathrm{S} / \mathrm{R}$ & $\mathbf{2 , 0 4} \mathbf{~ k g}$ & $1,85 \mathrm{~kg}$ & $1,81 \mathrm{~kg}$ & $1,87 \mathrm{~kg}$ \\
\hline Peso promedio & $\mathrm{S} / \mathrm{R}$ & $0,66 \mathrm{~kg}$ & $0,60 \mathrm{~kg}$ & $0,55 \mathrm{~kg}$ & $0,69 \mathrm{~kg}$ \\
\hline Peso mínimo & $\mathrm{S} / \mathrm{R}$ & $0,34 \mathrm{~kg}$ & $0,25 \mathrm{~kg}$ & $\mathbf{0 , 2 2} \mathbf{~ k g}$ & $0,45 \mathrm{~kg}$
\end{tabular}

Observación: solo se obtuvo el peso de las muestras entre el 2015 y 2018 $(\mathrm{S} / \mathrm{R}=$ Sin Registro $)$

La composición de talla de $L$. peru, el mayor número de especímenes estuvo entre los 24,9 y 42,1 cm (Fig.3), mientras que L. guttatus, estuvo entre los 28,4 y 45,2 cm (Fig.4).

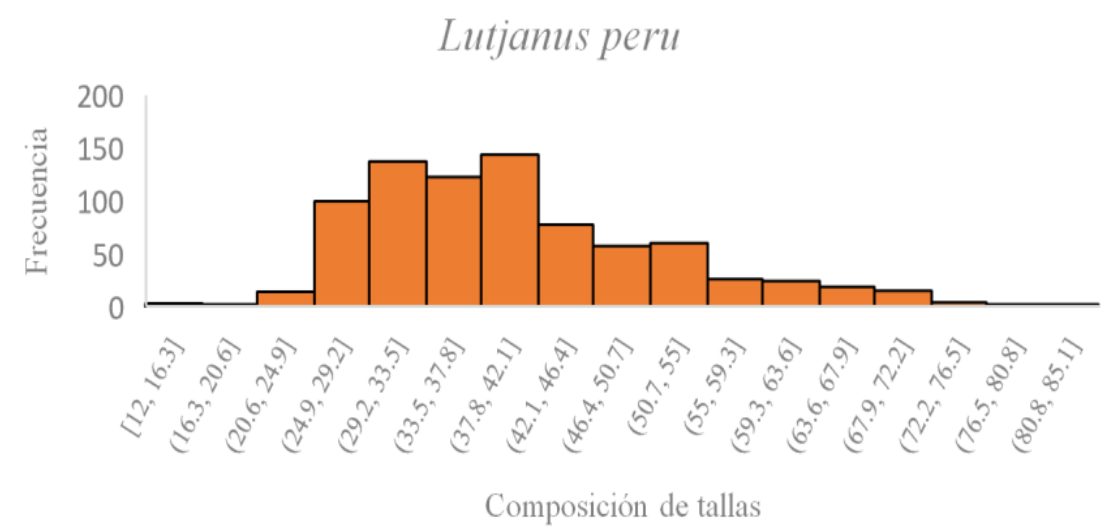

Fig. 3 Composición de tallas de Lutjanus peru en el Golfo de Montijo, periodo 2013-2018 


\section{Lutjanus guttatus}

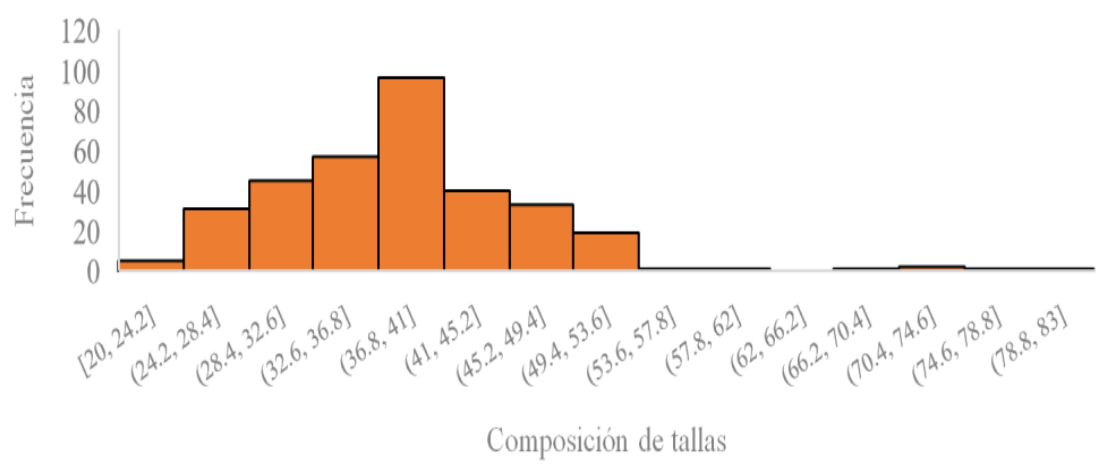

Fig. 4 Composición de tallas de Lutjanus guttatus en el Golfo de Montijo, period o 2013-2018

\section{Correlación talla- peso}

En $L$. peru el coeficiente de correlación $\mathrm{r}=0,76$ el coeficiente de determinación fue R2 =0,5705 y (Fig. 5); mientras que en L. guttatus el $r=0,73$ y R2=0,5338 (Fig. 6).

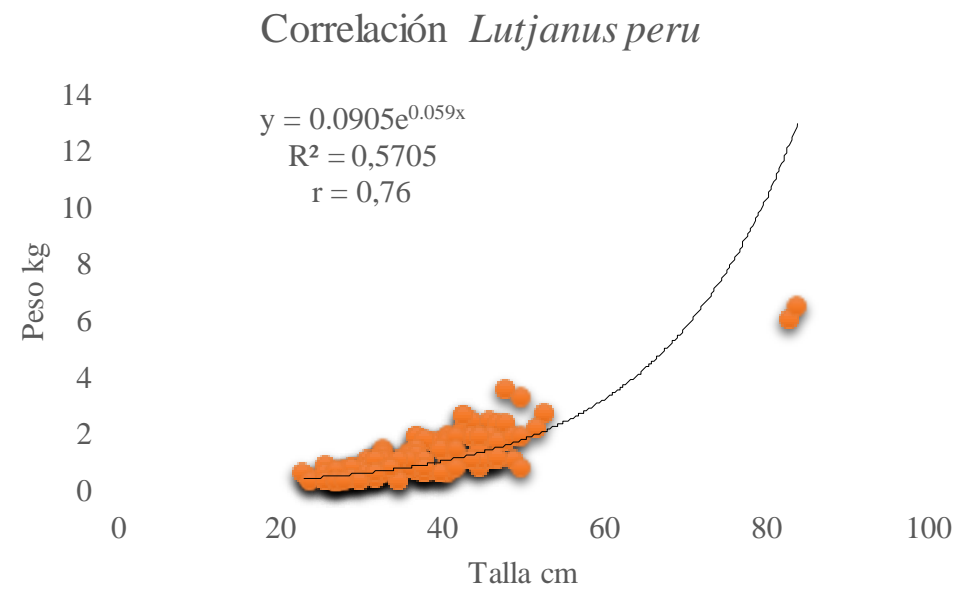

Fig. 5 Correlación talla-peso de la especie Lutjanus peru, pesca artesanal, Golfo de Montijo 


\section{Correlación Lutjanus guttatus}

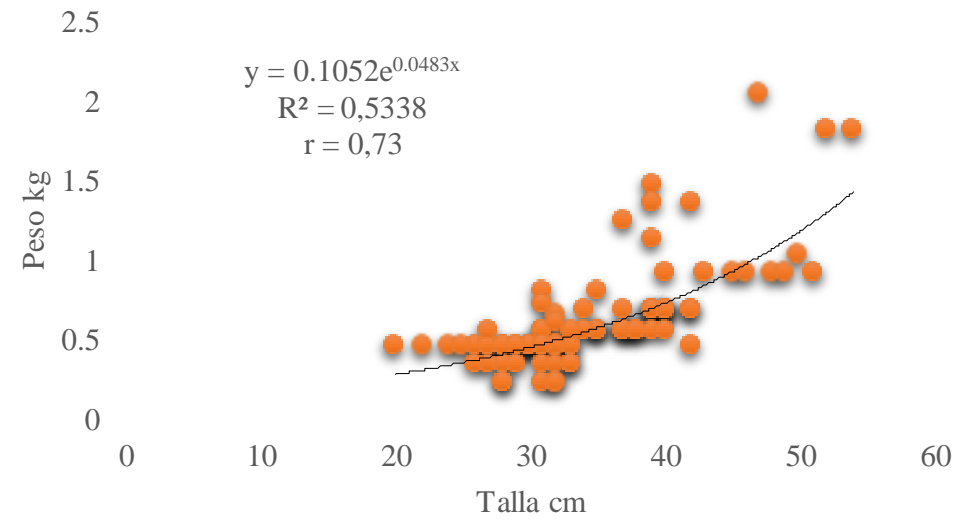

Fig. 6 Correlación talla-peso de la especie Lutjanus guttatus, pesca artesanal, Golfo de Montijo.

\section{DISCUSIÓN}

Las especies Lutjanus peru y L. guttatus, son las que mayor desembarque reportan en el Golfo de Montijo, y las que más demanda tienen desde el punto de vista alimentario.

En las costas de Guerrero, del Pacifico mexicano L. peru y L. guttatus, son las especies objetivo de capturas artesanal (Santamaría \& Chávez, 1999). Chiappa-Carrara et al. (2004) sostienen que son especies residentes y dominantes, se capturan de manera permanente a lo largo de todo el año, con los mismos artes y en los mismos caladeros, y son de gran importancia para las pesquerías ribereñas. Espino-Barr et al. (2003) las reportan para la costa de Colima y Ulloa et al. (2008) para el estado de Nayarit, México.

Barreto y Borda (2008), en Colombia reportan ambas especies en el Pacífico; en Costa Rica Araya et al. (2007) y Soto-Rojas et al. (2008) registran L. guttatus en el Golfo de Nicoya.

En el Pacífico panameño Durán Oliva (2004) reporta las especies $L$. peru y L. guttatus en Búcaro; Vega et al. (2016) en el Parque Nacional Coiba; Vergara (2017), en la bahía de Parita y Miranda y Sánchez (2018) registra L. guttatus en la misma zona. 
Estos autores coinciden con nuestro estudio, ya que, en el Golfo de Montijo, estas dos especies son las más explotadas, debido al aumento del esfuerzo pesquero dirigido a estas.

En el estado de Guerrero, México, entre las especies de mayor captura se destacan, por su valor y volumen, L. peru con 1000 t y L. guttatus con $650 \mathrm{t}$ al año (Rojas-Herrera, 1990). Por otro lado, de los recursos de escama que desembarca la flota artesanal en el Pacífico mexicano, $L$. peru es una de las especies más importantes por su valor comercial de acuerdo con el INP (2001). En México las artes de pesca utilizados en la captura de L. guttatus son la línea de fondo y el trasmallo (AmezcuaLinares, 1996; Espino-Barr et al. 2004; Rojo-Vázquez et al. 2008).

En el Pacífico colombiano según el boletín SEPEC (2014), indica que el 80,37 \% de los desembarcos son atribuidos a peces óseos, de los cuales la especie L. guttatus representa el $12 \%$. Mientras que, en Costa Rica, Araya et al. (2007), establecen que L. guttatus en las zonas 3 y 4 del Golfo de Nicoya, las capturas no presentan una tendencia clara, pero sí hay variaciones entre 1994 y 2005. Según Soto- Rojas et al. (2008) una de las pesquerías más importantes en el Golfo de Nicoya es la de $L$. guttatus, su captura se realiza con diversas artes y principalmente por la flota artesanal.

Los datos muestran que L. peru representa el 49,29\% de las capturas 2013-2018, manteniéndose en aumento el volumen anual, con máximo en el 2017 de $45895 \mathrm{~kg}$ y desciende en el 2018 en 32,86 \%. L. guttatus representa el $50,71 \%$ de la captura, en el año 2015 con $75488 \mathrm{~kg}$ y una reducción de $87,15 \%$ en el año 2018, con captura de $9699 \mathrm{~kg}$ que indica presión de la actividad pesquera.

De acuerdo con Vega et al. (2016), el volumen y el precio que se paga, por las especies de mayor valor comercial: L. peru, L. guttatus y $L$. argentiventris, explican su explotación en el Golfo de Montijo. Según Vergara (2017), en la Bahía de Parita la especie L. guttatus representa el 96,36 \% del total del desembarco, por otro lado, en el puerto El Agallito, esta especie constituye el $25 \%$ y en Boca de Parita el $15 \%$. 
En Panamá estas representan un volumen importante en la pesquería artesanal, aunque se ejerce presión de pesca, por lo cual el volumen disminuye a través de los años. De acuerdo con la FAO (2018) el estado de los recursos marinos monitoreados sigue deteriorándose, además, señala que la proporción de poblaciones de peces marinos pescados dentro de niveles biológicamente sostenibles ha mostrado una tendencia decreciente, desde 1974 a 2015 de 66, $9 \%$.

Ulloa et al. (2008) indican que L. peru, en el Pacífico mexicano, la talla mínima es de $16 \mathrm{~cm}$, con promedio $31,13 \mathrm{~cm}$ y la máxima $110,5 \mathrm{~cm}$; en L. guttatus varía de $97 \mathrm{~cm}$ a $19 \mathrm{~cm}$, con promedio $33,10 \mathrm{~cm}$; Barbosa Ortega (2016), en La Bahía de La Ventana, en Baja California Sur, $L$. peru, están entre 40,4 cm y 75,4 cm y media de 56,28cm. En el Pacífico colombiano Barreto y Borda (2008), registran tallas de L. peru de $21 \mathrm{~cm}$ la mínima, $99 \mathrm{~cm}$ máxima, promedio de $59,3 \mathrm{~cm}$.

Las tallas de L. peru, oscilan entre los 12 y $84 \mathrm{~cm}$, el 59,38\% están por debajo del promedio de captura $40,7 \mathrm{~cm}$ y el mayor número de ejemplares esta entre los 29,2 y 42,1 cm. En L. guttatus están entre los 20 y $79 \mathrm{~cm}$ y el $51,2 \%$ está por debajo de la talla promedio $38,5 \mathrm{~cm}$, registrándose el mayor número de ejemplares entre los 33 y $41 \mathrm{~cm}$. Pacheco (2003) reporta para L. peru que la LT media varía entre 32,57 $\mathrm{cm}$ en febrero y $66,15 \mathrm{~cm}$. en abril.

Las tallas promedio de L. peru coinciden con las reportadas por Vega et al. (2016) quienes señalan que en el Parque Nacional Coiba, hay un alto porcentaje de capturas de individuos, de menos de $40 \mathrm{~cm}$ de LT, la talla media es de $42 \mathrm{~cm}$, con una estructura desviada hacia tallas pequeñas. Por otro lado, L. guttatus presenta mayor frecuencia de individuos por encima de $35 \mathrm{~cm}$, mientras que la talla media de captura se estima en $40,5 \mathrm{~cm}$.

Según Durán Oliva (2004) L. peru reportan tallas entre los 20,5 cm y $64,8 \mathrm{~cm}$ en ambos sexos y peso entre $85 \mathrm{~g}$ y $3,62 \mathrm{~kg}$; en $L$. guttatus las tallas fluctuaron entre $20,4 \mathrm{~cm}$ y $61 \mathrm{~cm}$, y peso entre $113 \mathrm{~g} \mathrm{y} 27,22 \mathrm{~kg}$. Vergara (2017) en el puerto El Agallito reporta tallas de L. guttatus entre los 21 y $49 \mathrm{~cm}$ con promedio de $31,01 \mathrm{~cm}$ y peso entre los $100 \mathrm{~g}$ y 1,13 $\mathrm{kg}$ con un promedio de 318,25 g; para el puerto el Boca de Parita entre 
los $21,5 \mathrm{y} 45 \mathrm{~cm}$, con un promedio de $28,19 \mathrm{~cm}$; y peso entre los $91 \mathrm{~g} \mathrm{y}$ $1,36 \mathrm{~kg}$, con promedio de 416,25 g.

En este estudio el ámbito de talla promedio y el de pesos en ambas especies es mayor a los reportados por Durán Oliva (2004) en Búcaro y Vergara (2017) en la Bahía de Parita, pero similares a los presentados para el Pacífico de México y Colombia.

Los porcentajes más altos observados corresponden a peces por debajo del promedio, eso es un indicativo de que se captura peces de tallas medianas a pequeñas, aun cuando el arte de pesca utilizado busca la selectividad, cuya captura pone en peligro la reproducción y la sostenibilidad de las especies mencionadas.

Autores como Gallardo-Cabello et al. (2010) señalan que la longitud corporal de $L$. peru en su primera madurez sexual es de $25,45 \mathrm{~cm}$ y su longevidad de 12 años; el 65,84 \% de la pesca en Bahía Bufadero, México, es de organismos sexualmente inmaduros, por lo que se propone como talla mínima de captura $45 \mathrm{~cm}$ (edad 3 años).

Dichas variaciones en el tamaño de captura pueden deberse a la malas práctica de pesca por parte de los pescadores. Según la FAO (2018) los efectos del cambio climático se sienten por todo el mundo, con consecuencias para los sistemas marinos a través de la red alimentaria, incluidas especies que sustentan las pesquerías.

En el Golfo de Nicoya, Pacífico de Costa Rica, Soto-Rojas et al. (2008), indican una relación talla-peso para L. guttatus de Pt $=0,019$ LT 2,867 $r=0,996$ en organismos enteros y $\mathrm{Pe}=0,017 \mathrm{LT} 2,872 \mathrm{r}=0,989$, en eviscerado. Sin embargo, en el Pacífico mexicano Barbosa Ortega (2016) en La Bahía de La Ventana, Baja California Sur reporta el r = 0,978 para la especie L. peru.

En Búcaro, Pacífico panameño, Durán Oliva (2004) señala que para la especie L. peru, en la relación longitud-peso se obtuvo un coeficiente de correlación $\mathrm{r}=0,97$; mientras que para la especie L. guttatus, fue de r = 0,94. En la Bahía de Parita, Miranda y Sánchez (2018), señalan que la relación entre la longitud y peso está dada por la ecuación: PT $=0,042$ LT 2,67, r =0,89, y el valor del exponente fue diferente a 3 , lo que indica un crecimiento alométrico negativo. 
Para el Golfo de Montijo, el valor del coeficiente de correlación para cada especie mostró tener un grado de relación aceptable con tendencia al valor de uno, que indica una correlación alta. La diferencia en las correlaciones entre este estudio y la de los demás se debe al hecho de que son poblaciones diferentes sometidas a condiciones ambientales distintas.

Si bien es cierto, los resultados obtenidos coinciden con los obtenidos por otros autores, el recurso pesquero estudiado, se encuentran bajo una presión pesquera permanente, como lo indica el volumen de captura.

Aunado a ello, el alto porcentaje de peces con tallas menores a los valores promedios, confirman capturas de ejemplares de tallas medias a pequeñas.

Según la Gaceta Oficial Digital No. 28365-B, Decreto Ejecutivo N 126 septiembre de 2017 se regula el uso del Arte de Pesca Palangre.

En ese sentido, infringir las normas que regulan dicha actividad generan problemas para la sostenibilidad pesquera, ya que al capturar peces juveniles cortan su ciclo biológico y afecta la reproducción de dichas especies, lo que puede causar escases del recurso y poner en peligro la seguridad alimentaria.

\section{CONCLUSIONES}

Se reportó la talla máxima $84 \mathrm{~cm}$ para Lutjanus peru y $79 \mathrm{~cm}$ para $L$. guttatus, con mayores capturas entre los $24,9 \mathrm{~cm}-42,1 \mathrm{~cm}$ y $28,4 \mathrm{~cm}-$ $45,2 \mathrm{~cm}$ respectivamente.

Las capturas en el Golfo de Montijo para Lutjanus peru y L. guttatus representa $49,29 \%$ y 50,71\% del volumen total comprendido entre los años 2013 y 2018.

La relación talla-peso para ambas especies resultó ser alométrica negativa, con alto grado de correlación. 
El estudio de esta pesquería aporta valiosa información que contribuye a un mejor entendimiento del recurso, que garantiza el equilibrio de la pesca, para una mejor toma de decisiones que conlleve a la sostenibilidad.

\section{AGRADECIMIENTOS}

A la Autoridad de los Recursos Acuáticos de Panamá (ARAP) por brindar el apoyo y permitirnos trabajar con la base de datos de peces, pesca artesanal en el Pacífico veragüense y por la autorización para la publicación del presente artículo.

\section{REFERENCIAS}

Amezcua-Linares, F. (1996). Peces demersales de la plataforma continental del Pacífico Central de México. Instituto de Ciencias de Mar y Limnología, UNAM, Comisión Nacional para el Conocimiento y Uso de la Biodiversidad, México, D.F., México.

ARAP. (2011). Guía para la Identificación de Peces de Interés Comercial para el Pacífico de Panamá. Dirección de Investigación y Desarrollo. Documento Técnico de Pesca. Ciudad de Panamá, Panamá.

Araya, H., A. Vásquez, B. Marín, J. A. Palacios, R. Soto Rojas, F. Mejía-Arana, Y. Shimazu y Hiramatsu, K. (2007). Reporte no.1/2007 del Comité de Evaluación de Poblaciones: Reporte del Manejo de los Recursos Pesqueros en el Golfo de Nicoya. In: Proyecto Manejo Sostenible de la Pesquería en el Golfo de Nicoya: Presentación de conclusiones y recomendaciones. UNA-JICA-INCOPESCA. Puntarenas, Costa Rica.

Barbosa Ortega, W.A. (2016). Estructura de las capturas y longitud de madurez del huachinango Lutjanus peru (perciformes: Lutjanidae), en la Bahía de la Ventana, B.C.S., México. [Tesis de Maestría, INSTITUTO POLITECNICO NACIONAL, CENTRO INTERDISCIPLINARIO DE CIENCIAS MARINAS]. 
Barreto, C.G. y Borda, C.A. (2008). Propuesta Técnica para la definición de Cuotas Globales de Pesca para Colombia, Vigencia 2009.

Cámara A., R., F. Díaz Olmo, J.R. Martínez B., M. del C. Morón M., C. Gómez-Ponce, E. Tabares, y Vega, A.J. (2004). Directrices de gestión para la conservación y desarrollo integral de un humedal centroamericano: Golfo de Montijo (Litoral del Pacífico de Panamá). Cooperación Española, Fundación Demuca, MEF-ANAM.

Chiappa-Carrara, X., Rojas-Herrera, A.A. y Mascaró, M. (2004). Coexistencia de Lutjanus peru y Lutjanus guttatus (Pisces: Lutjanidae) en la costa de Guerrero, México: relación con la variación temporal en el reclutamiento. Rev. biol. Trop, 52(1),177-185.

CREHO. (2009). Centro Regional Ramsar para la Capacitación e Investigación sobre Humedales para el hemisferio occidental. Inventario de los humedales continentales y costeros de la República de Panamá. Flores De G., E., Gallardo, M., Núñez, E. (eds.). Panamá.

Durán Oliva, I. L. (2004). Diagnóstico pesquero y socioeconómico de la pesca artesanal en la comunidad de Búcaro, Rep. De Panamá: con recomendaciones para el manejo de la familia Lutjanidae. [Tesis de Maestría, Universidad de Costa Rica].

Espino Barr, E., Cruz Romero, M. y García Boa, A. (2003). Peces marinos con valor comercial de la costa de Colima, México. CONABIO-INP-CRIP Manzanillo.

Espino-Barr, E., Cabral-Solís, E.G., García-Boa, A. y Puente-Gómez, M. (2004). Especies marinas con valores comerciales de la costa de Jalisco, México. CRIP, Manzanillo, México.

FAO. (2016). El estado mundial de la pesca y la acuicultura. Contribución a la seguridad alimentaria y la nutrición para todos. Roma.

FAO. (2018). Impactos del cambio climático en la pesca y la acuicultura: Síntesis de los conocimientos y las opciones de adaptación y mitigación actuales. Resumen del Documento Técnico de Pesca y Acuicultura de la FAO no. 627. Roma. 
Decreto Ejecutivo $\mathrm{N}^{\circ} 126$ de 2017. Que regula la licencia de pesca para naves de servicio interior que utilizan el arte de pesca denominado palangre en las aguas jurisdiccionales de la República de Panamá y dicta otras disposiciones. Septiembre de 2017. Gaceta Oficial Digital No. 28365-B.

Gallardo-Cabello, M., Sarabia-Méndez, M., Espino-Barr, E. y AnisladoTolentino, V. (2010). Biological aspects of Lutjanus peru in Bufadero Bay, Michoacán, México: growth, reproduction and condition factors. Revista de biología marina y oceanografía, 45(2), 205-215. https://dx.doi.org/10.4067/S0718-19572010000200002

INP. (2001). Sustentabilidad y Pesca Responsable en México: Evaluación y Manejo. Instituto Nacional de la Pesca (INP). Secretaría de Agricultura, Ganadería, Desarrollo Rural, Pesca y Alimentación, México.

Lucano-Ramírez, G., Ruiz-Ramírez, S., González-Sansón, G. y Ceballos-Vázquez, B.P. (2012). Biología reproductiva del pargo Lutjanus inermis (Perciformes: Lutjanidae), en el Pacífico central mexicano. Rev. Biol. Trop. 60 (1), 393-403.

Maté J. L. (2005). Análisis de la situación de la pesca en los golfos de CHIRIQUÍ y de MONTIJO. The Natural Conservancy, Instituto Smithsonian de Investigaciones Tropicales.

Miranda, H. y Sánchez, E. (2018). Análisis de talla, contenido estomacal y gonadal en las principales especies de interés comercial capturado en La Bahía de Parita, Panamá. Revista Científica Guacamaya, 3(1), 4560.

Pacheco, L. (2003). Ciclo reproductivo de la especie Lutjanus peru (Nichols y Murphy, 1922), aspectos microscópicos del crecimiento y desarrollo de los ovocitos. [Tesis de Licenciatura, Universidad de Panamá].

Robertson, D. R. y Allen, G. R. (2008). Peces Costeros del Pacífico Oriental Tropical: Sistema de Información en línea. Versión 1.0 (2008). Instituto Smithsonian de Investigaciones Tropicales, Balboa, República de Panamá. www.neotropicalfishes.org/sftep, www.stri.org/sftep 
Robertson, D. R. y Allen, G. R. (2015). Peces Costeros del Pacífico Oriental Tropical: Sistema de Información en línea. Versión 2.0 Instituto Smithsonian de Investigaciones Tropicales, Balboa, República de Panamá.

Rojas-Herrera, A.A. (1990). Análisis de las capturas en las pesquerías del estado de Guerrero, México. Mem. VII Congreso Nacional de Oceanografía, Mazatlán, Sin. Mex.

Rojo-Vázquez, J.A., C. Quiñonez-Velázquez, H.A. Echavarría-Heras, G. Lucano-Ramírez, E. Godínez Domínguez, S. Ruiz-Ramírez, V.H. Galván-Piña y Sosa-Nishizaki, O. (2008). The fish species composition and variation of catch from the small-scale gillnet fishery before, during and after the 1997-1998 ENSO event, central Mexican Pacific. Rev. Biol. Trop. 56(1),133-152.

Santamaría, A. y Chávez, E.A. (1999). Evaluación de la pesquería de Lutjanus peru (Pisces: Lutjanidae) de Guerrero, México. Rev. biol. Trop, 47(3),571-580.

SEPEC. (2014). Boletín estadístico. Enero - agosto de 2013. Autoridad Nacional de Acuicultura y Pesca - AUNAP.

Soto-Rojas, R.L., Mejía-Arana, F., Palacios, J.A. y Hiramatsu, K. (2008). Reproducción y crecimiento del pargo mancha Lutjanus guttatus (Pisces: Lutjanidae) en el Golfo de Nicoya. Estación de Biología Marina, Escuela de Ciencias Biológicas, Universidad Nacional, Puntarenas, Costa Rica.

Ulloa Ramírez, P.A., Patiño Valencia, J.L. Guevara Rascado, M. De L., Hernández Ventura, S., Sánchez Regalado, R. y Pérez Velázquez, A. (2008). Peces Marinos de valor comercial del Estado De Nayarit, México. Instituto Nacional de Pesca Centro Regional de Investigación Pesquera Bahía de Banderas, Nayarit.

Vega, A.J. y Villareal, N. (2003). Peces asociados a Arrecifes y manglares en El Parque Nacional Coiba. Tecnociencia, 5(1), 65-76.

Vega, A.J., Y. A. Robles P. y Maté, J. L. (2016). La pesca artesanal en el Parque Nacional Coiba y zona de influencia. Biología y pesquería de 
sus principales recursos, con recomendaciones de manejo. Fundación MarViva, Ciudad de Panamá, Panamá.

Vergara, Y. (2017). Caracterización de la pesca artesanal en la Bahía de Parita y análisis de desembarque en los puertos de Boca Parita y el Agallito, provincia de Herrera, Panamá. [Tesis de Maestría, Universidad de Panamá]. 